\title{
Modeling COVID-19 and Its Impacts on U.S. Immigration and Customs Enforcement (ICE) Detention Facilities, 2020
}

\author{
Michael Irvine • Daniel Coombs • Julianne Skarha • \\ Brandon del Pozo • Josiah Rich • Faye Taxman • Traci \\ C. Green
}

Published online: 15 May 2020

(C) The New York Academy of Medicine 2020

\begin{abstract}
U.S. Immigration and Customs Enforcement (ICE) facilities house thousands of undocumented immigrants in environments discordant with the public health recommendations to reduce the transmission of 2019 novel coronavirus (COVID-19). Using ICE detainee population data obtained from the ICE Enforcement and Removal Operations (ERO) website as of March 2, 2020, we implemented a simple stochastic susceptibleexposed-infected-recovered model to estimate the rate of COVID-19 transmission within 111 ICE detention facilities and then examined impacts on regional hospital intensive care unit (ICU) capacity. Models considered
\end{abstract}

Daniel Coombs and Michael Irvine are co-first authors.

Electronic supplementary material The online version of this article (https://doi.org/10.1007/s11524-020-00441-x) contains supplementary material, which is available to authorized users.

\section{Irvine}

British Columbia Children's Hospital Research Institute,

Vancouver, British Columbia, Canada

D. Coombs

Department of Mathematics and Institute of Applied Mathematics, University of British Columbia, Vancouver, British Columbia, Canada

J. Skarha $\cdot$ B. del Pozo

Department of Epidemiology, Brown University School of Public Health, Box G-S121-3, Providence, RI 02912, USA

J. Rich

Brown University School of Medicine, The Miriam Hospital, Providence, RI, USA three scenarios of transmission (optimistic, moderate, pessimistic) over 30-, 60-, and 90-day time horizons across a range of facility sizes. We found that $72 \%$ of individuals are expected to be infected by day 90 under the optimistic scenario $(\mathrm{R} 0=2.5)$, while nearly $100 \%$ of individuals are expected to be infected by day 90 under a more pessimistic $(\mathrm{R} 0=7)$ scenario. Although asynchronous outbreaks are more likely, day 90 estimates provide an approximation of total positive cases after all ICE facility outbreaks. We determined that, in the most optimistic scenario, coronavirus outbreaks among a minimum of 65 ICE facilities (59\%) would overwhelm ICU

\section{F. Taxman}

Center for Advancing Correctional Excellence, Department of Criminology, Law and Society, George Mason University, Fairfax, VA, USA

\section{T. C. Green}

Opioid Policy Research Collaborative, Heller School for Social Policy and Management, Brandeis University, 415 South Main St. MS 035, Waltham, MA 02454-9110, USA

T. C. Green $(\bowtie)$

Departments of Emergency Medicine and Epidemiology, Brown Schools of Medicine and Public Health, Providence, RI, USA e-mail: Traci.c.green@gmail.com 
beds within a 10-mile radius and outbreaks among a minimum of 8 ICE facilities (7\%) would overwhelm local ICU beds within a 50 -mile radius over a 90 -day period, provided every ICU bed was made available for sick detainees. As policymakers seek to rapidly implement interventions that ensure the continued availability of life-saving medical resources across the USA, they may be overlooking the pressing need to slow the spread of COVID-19 infection in ICE's detention facilities. Preventing the rapid spread necessitates intervention measures such as granting ICE detainees widespread release from an unsafe environment by returning them to the community.

Keywords Immigration · Detention · COVID-19 . coronavirus $\cdot$ Migrant health

\section{Background}

The USA operates the largest immigrant detention system in the world [1], with over 140,000 immigrants arrested in 2019 or nearly 383 people a day. The conditions of confinement at U.S. Immigration and Customs Enforcement (ICE) facilities are designed to control and closely surveil the population. This setting is discordant with public health guidance aiming to reduce the transmission of 2019 novel coronavirus disease (COVID-19), especially including advice around physical distancing and frequent hand washing. Furthermore, because detained immigrants are not housed individually or in cells as is true in many prison settings, detainees have more contacts with one another and with staff, thereby contributing to faster spread of infection than in correctional facilities. On March 24, 2020, ICE recorded its first documented COVID-19 case: a 31-yearold immigrant at Bergen County Jail in New Jersey. This case, documented less than a week after a correctional staff member at the facility also tested positive [2], underscores the potential of this virus to rapidly spread in enclosed environments and the potential for COVID19 outbreaks in other detention facilities. As of April 16, 2020, ICE reported that 100 detainees had tested positive for COVID-19, across 13 states [3].

COVID-19 is a respiratory illness that most severely affects individuals over the age of 65 and individuals with underlying health conditions, such as those who are immunocompromised, have asthma, or suffer from diabetes. COVID-19 can lead to severe conditions, including breathing difficulties and lung damage, hospitalization, admission to a hospital intensive care unit (ICU), and death. Although public data on the health status and underlying health conditions of individuals detained in ICE detention facilities are limited, numerous reports document insufficient medical care as detailed in the lay press [4], whistleblower complaints [5, 6], and a Department of Homeland Security report [7]. The poor medical care of detainees includes missed diagnoses and incorrect vaccine doses. Additionally, the literature documenting the health of undocumented individuals indicates they are more likely than the general population to be uninsured, have had poor prenatal care, and face stressors such as stigma, discrimination, and fear of deportation - all factors that negatively impact physical and mental health [8-10].

In response to mounting concern about the potential for a COVID-19 outbreak among this highly vulnerable population of individuals held in detention facilities, ICE has reportedly implemented several measures aimed at reducing the risk of virus transmission. Such measures are said to include suspending social visitation and releasing a small subset of individuals who are determined to be a low "threat to public safety, flight risk, and national security" and have underlying health conditions that may cause acute complications upon infection [3]. However, outbreaks at correctional facilities across the country suggest that these measures are insufficient to prevent COVID-19 infections within enclosed environments. Cook County Jail, for example, was the largest singlesite source of COVID-19 cases in the nation. From March 22 to April 12, 2020, the number of cases at the jail grew from one incarcerated individual to over 300 known cases among prisoners and over 180 among correctional staff. On April 20, 2020, the Marion Correctional Institution, an Ohio prison, reported a 78\% COVID-19 infection rate: 1950 inmates and 154 staff had been diagnosed with the virus there [11]. The prison reported its first case just three weeks prior on March 29th [12], underscoring the fast pace of transmission in such environments.

As COVID-19 continues to spread in communities, and inevitably into the nation's detention centers, it is critical that we understand the US healthcare system's capacity to care for a large influx of patients who require critical care. In the present study, we estimate the number of COVID-19 cases likely to occur within ICE detention facilities, using varying levels of virus 
transmissibility, and examine the capacity of local hospitals to care for additional patient ICE detainees over 30-, 60-, and 90-day time horizons.

\section{Methods}

Model

We implemented a simple stochastic susceptibleexposed-infected-recovered (SEIR) model. In this model, individuals are initially susceptible (S) to COVID-19 infection and become infected and transition to an exposed class (E) at a rate dependent on the reproductive number, known as the R0, and the proportion of individuals who are currently infectious (I). Individuals in the exposed class then transition to an infectious state (I) after a latent period and, finally, to a recovered state (R) at a constant recovery rate. The SEIR model and related variants support the vast majority of COVID-19 modeling studies around the world at present.

The transition states of the model are as follows:

$\mathrm{S} \rightarrow \mathrm{E}$ at rate $\beta \mathrm{SI} / N$

$\mathrm{E} \rightarrow \mathrm{I}$ at rate $\sigma$

$\mathrm{I} \rightarrow \mathrm{R}$ at rate $\gamma$

where $N$ is the fixed population size, $\beta$ is the transmissibility, $1 / \sigma$ is the incubation period, and $1 / \gamma$ is the recovery period. All events are assumed to be Poissondistributed, and a Tau-leaping method was implemented to draw realizations of the epidemic process. For each scenario, 1000 replicates were drawn. To prevent stochastic burnout, we performed simulations using an initial condition of five infectious detainees. Numbers of cases are reported as medians with confidence intervals arising directly from multiple realizations of the stochastic model. In addition to estimating the number of infected individuals, our simulations also show the timing and size of the peak of the epidemic (see Supplementary Information).

The estimated proportion of infected people requiring hospitalization and/or ICU admissions during our simulated epidemics is calculated from the cumulative number of infectious individuals over 30-, 60-, and 90day time horizons. The numbers of individuals requiring hospitalization or ICU admission were estimated based on the age distribution of people within the facility (see below), multiplied by Centers for Disease Control and Prevention (CDC) estimates of the fraction of infected people within defined age classes requiring treatment [13]. We used the minimum rate for each age group in our calculations.

\section{Initial Conditions}

On day 0 of the epidemic, five individuals are assumed to be infected (in state I), with the rest being susceptible for a range of fixed population sizes chosen to be reflective of facility sizes: 50, 100, 500, and 1000 .

\section{Parameterization}

Given the current unknowns in the rate of spread of COVID-19 in detention centers, epidemiological parameters were based on estimates of an outbreak on cruise ships, representing a controlled environment with a high rate of contact between individuals. $\mathrm{R} 0$ is the expected number of cases directly generated by one case in a population where all individuals are susceptible to infection and is a product of the rate of contact between individuals, the probability of infection per contact, and the average time an individual is infectious. Estimates for the R0 based on the Diamond Princess cruise ship outbreak range from 2.28 (95\% CI 2.06-2.52) [14] to as high as 14.8 [15]. Outbreaks have also been documented onboard several other cruise ships and navy ships, including the aircraft carrier USS Theodore Roosevelt, where nine crew members are currently hospitalized and one has died. Studies based on these confined settings for viral spread will provide additional information for future studies. Drawing from the published estimates, we studied three scenarios ranging from a most optimistic to most pessimistic estimate of the R0 as 2.5, 3.5, and 7 for low, medium, and high R0 scenarios, respectively. The incubation and infectious periods were estimated to be 6.4 days [16] and 7 days [17], respectively.

\section{Population Data}

ICE detainee population data were downloaded directly from the Department of Homeland Security Enforcement and Removal Operations (ERO) website. The average number of detainees between October 1, 2019 and March 2, 2020 was used to approximate the current number of people detained at each detention facility and summed to approximate the total number of people currently detained by ICE. To our knowledge, these are the most up-to-date estimates of detainees per 
facility. We excluded facilities with less than 25 people to meet the minimum population requirement for stability of estimates in the COVID-19 model. ICE currently withholds the age of arrest for detainees [18]; thus, we used the age distribution provided by the Freedom for Immigrants, an advocacy organization that has interviewed thousands of people in immigration detention and reported age demographics in April 2019 [19].

We determined ICU capacity using the Definitive Healthcare USA Hospital Bed data [20]. Definitive Healthcare is a leading provider of data on healthcare organizations throughout the USA and has made these data freely available during the COVID-19 crisis. We used the version of this data updated on April 3, 2020. The Definitive Healthcare USA group also created an ICU capacity predictor tool [21]. This tool combines ICU hospital bed and ventilator availability/utilization data, Census Bureau population density, demographic information, and New York Times COVID-19 case data to determine the ICU capacity per county. We pulled this information for each county with an ICE facility. We then determined the ICU capacity for each ICE facility in a geospatial analysis program (ArcGIS; ESRI, Redlands, CA). We geocoded the ICE facilities and incorporated the Definitive Healthcare USA hospital bed data for analysis. We used the geospatial Buffer tool to create a 10-mile and 50-mile buffer around each ICE facility (Buffer, ArcGIS). Radii of 10 and 50 miles were selected as healthcare service catchment areas within which detention facilities may reasonably be expected to transport for patient care [22]. We used the Spatial Join tool from the spatial analysis toolbar to pull ICU bed information from the hospitals that were located in the 10-mile and 50-mile ICE facility buffers (Spatial Join, ArcGIS). We summed the ICU bed total per ICE facility (R, version 3.3) and applied the Definitive Healthcare USA ICU capacity predictor to determine the final number of ICU beds available to each ICE facility.

\section{Results}

Data for the models of COVID-19 infections, hospitalizations, and ICU admissions considered the average detention population from October 1, 2019 to March 2, 2020 in 111 facilities across 32 states. The number of detainees in each facility during this period ranged from 25 to 1600 (average 381, median 234 detainees), with a total of 42,435 individuals detained. Thirty-two percent $(n=35)$ of the included facilities hold only ICE detainees, and 68\% $(n=76)$ are city or county correctional facilities that hold ICE detainees and other individuals who are incarcerated. Although sex data were not available at the individual level, we obtained facility-level sex data. There were 577 women held in two female-only facilities; 15,560 individuals were held in 43 maleonly facilities; and 26,208 detainees were in facilities that held both females and males.

Based on 2019 data provided by Freedom for Immigrants, the majority of immigrants detained by ICE are between 26 and 35 years old, where the median age is 30 [19]. Country of origin for detainees included in this analysis was not available; however, a 2019 report by the U.S. Government Accountability Office found that the majority of detentions from 2015 to 2018 were males from Mexico, Guatemala, El Salvador, and Honduras, without a previous arrest or conviction record [23].

\section{Number of Infections over Time in Single-Facility Outbreaks}

Table 1 shows the estimated number of COVID-19 cases across the three R0 scenarios over 30-, 60-, and 90-day time horizons, for ICE facilities holding 50, 100, 500, and 1000 detainees. In a facility holding 1000 people, under the most optimistic scenario $(\mathrm{R} 0=2.5$ ), a median of 62 individuals are expected to have been infected by day 30 , 363 by day 60 , and 722 by day 90 . Under the least optimistic scenario of $\mathrm{R} 0=7$, the number of cumulative COVID-19 cases is modeled to increase from 732 by day 30 , to 989 by day 60 , to 993 by day 90 . The day 90 estimates, corresponding to $76 \%, 80 \%, 77 \%$, and $72 \%$ of all detainees in facilities of size 50, 100, 500, and 1000 detainees, give a sense of the total population of ICE detainees who are likely to be infected by the end of an ICE facility outbreak under the most optimistic scenario of $\mathrm{R} 0=2.5$ (Fig. 1). Regarding the timing and size of the peak of the epidemic, as one would predict, simulated epidemics within small facilities reach their peak earlier than those within large facilities (for example, on day 19 for facilities of 50 detainees vs day 69 for facilities of 1000 detainees with R0 $=3.5$; see Supplemental Materials).

Healthcare Service Utilization during Facility Outbreaks

Under the most optimistic scenario $(\mathrm{R} 0=2.5)$, and imagining synchronized epidemics across all facilities, 
Table 1 For different sized facilities and estimates of the basic reproduction number $\mathrm{R} 0$, estimated median (and $95 \%$ confidence intervals) aggregate number of infected detainees, to 30,60 , and
90 days after the outbreak began. Median hospitalization and ICU admission numbers (to day 90) are also shown

\begin{tabular}{|c|c|c|c|c|c|}
\hline \multirow[t]{2}{*}{ Facility size } & \multicolumn{3}{|c|}{ Median number (95\% CI) COVID-19 positive } & \multicolumn{2}{|c|}{ Median number ( $95 \% \mathrm{CI}), 90$ days } \\
\hline & 30 days & 60 days & 90 days & Hospitalizations & ICU admissions \\
\hline \multicolumn{6}{|l|}{$\mathrm{R} 0=2.5$} \\
\hline 50 & $25(7,36)$ & $36(11,43)$ & $38(11,44)$ & 6 & 1 \\
\hline 100 & $36(7,62)$ & $71(15,87)$ & $80(19,90)$ & 13 & 2 \\
\hline 500 & $60(12,128)$ & $258(46,366)$ & $386(181,437)$ & 58 & 9 \\
\hline 1000 & $494(491,495)$ & $363(35,605)$ & $722(203,834)$ & 114 & 17 \\
\hline \multicolumn{6}{|l|}{$\mathrm{R} 0=3.5$} \\
\hline 50 & $33(16,42)$ & $42(32,45)$ & $43(35,45)$ & 7 & 1 \\
\hline 100 & $59(24,80)$ & $88(69,94)$ & $90(79,94)$ & 15 & 2 \\
\hline 500 & $136(36,232)$ & $422(277,460)$ & $466(441,480)$ & 70 & 11 \\
\hline 1000 & $163(40,309)$ & $792(491,884)$ & $933(891,956)$ & 148 & 22 \\
\hline \multicolumn{6}{|l|}{$\mathrm{R} 0=7$} \\
\hline 50 & $43(38,45)$ & $45(44,45)$ & $45(44,45)$ & 7 & 1 \\
\hline 100 & $89(77,93)$ & $95(93,95)$ & $95(93,95)$ & 16 & 2 \\
\hline 500 & $407(310,444)$ & $493(489,495)$ & $494(491,495)$ & 75 & 11 \\
\hline 1000 & $732(519,823)$ & $989(982,994)$ & $993(989,995)$ & 157 & 24 \\
\hline
\end{tabular}

CI confidence interval

a median of 917 detainees system-wide will become infected and require hospitalization within the first 30 days, growing to an aggregate of 3129 patients by day 60 and 4909 by day 90 . Averaging across the total ICE system, this represents $11.6 \%$ of all detainees. The high hospitalization rate is driven by sustained

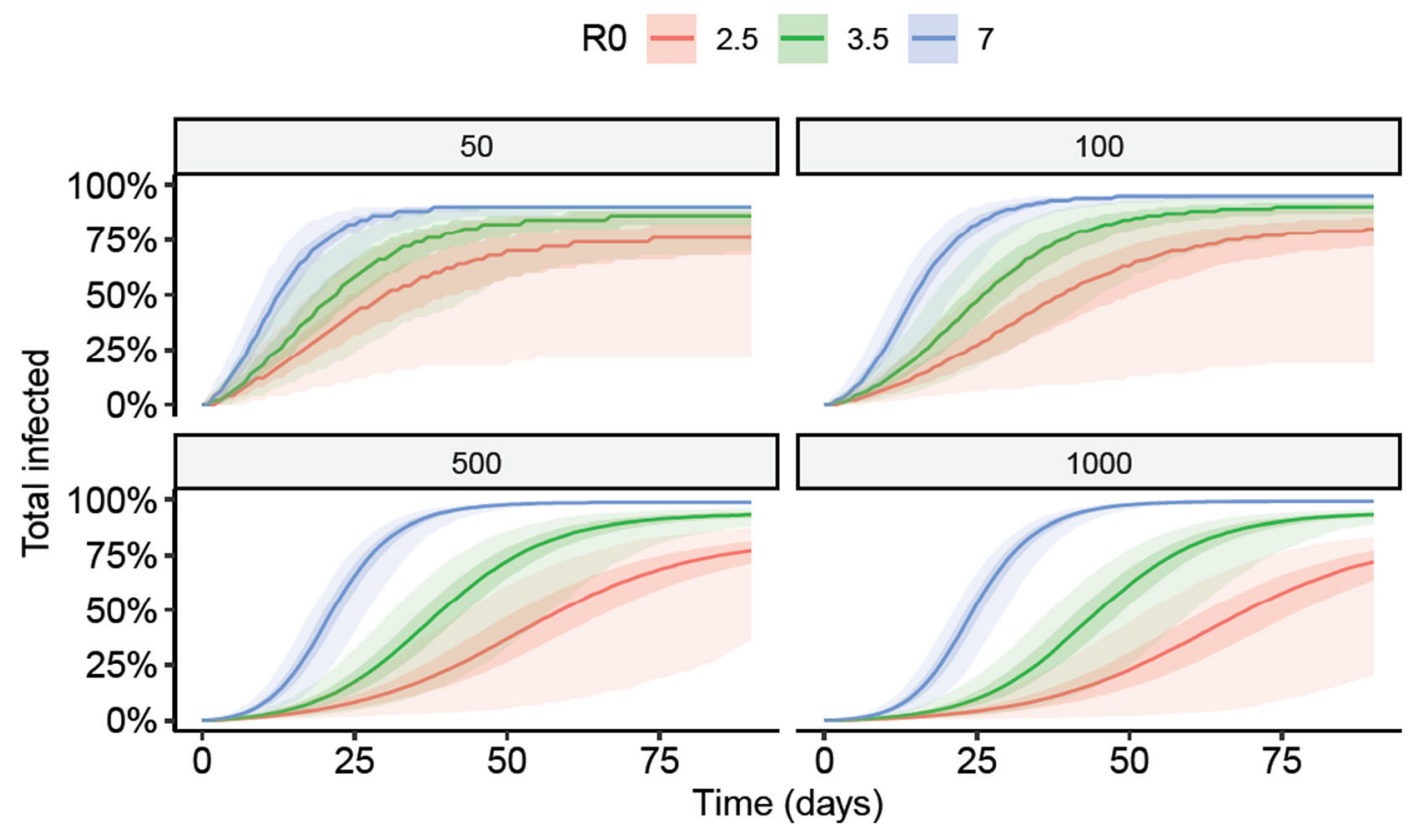

Fig. 1 Proportion of population infected with COVID-19 over time by R0 value and facility size 
transmission leading to very high fractions of detainees becoming infected, in the absence of robust and sustained countermeasures. The corresponding numbers for ICE facilities of different sizes at day 90 are given in Table 1. Under the pessimistic scenario of $\mathrm{R} 0=7$, and imagining synchronized epidemics across all facilities, a median of 5145 detainees system-wide will become infected and require hospitalization within the first 30 days, growing to an aggregate of 6391 patients by day 60 and 6408 patients by day 90. Averaging across the total ICE system, this represents $15.1 \%$ of all detainees. Concretely, in a facility housing 1000 detainees, the estimated median number of people infected and requiring hospitalization by day 30 ranges from 10 $(\mathrm{R} 0=2.5)$ to $26(\mathrm{R} 0=3.5)$ and $116(\mathrm{R} 0=7)$ and by day 90 ranges from $114(\mathrm{R} 0=2.5)$ to $148(\mathrm{R} 0=3.5)$ and $157(\mathrm{R} 0=7)$.

Focusing now on estimating ICU admissions, we estimate that with $\mathrm{R} 0=2.5$, and again imagining that all epidemics are synchronized, there will be a median of 139 detainees infected who will require ICU admission after 30 days, growing to an aggregate of 475 patients by day 60 and 745 patients by day 90 (approximately $1.8 \%$ of the total population). Under the pessimistic scenario of $\mathrm{R} 0=7$, the corresponding numbers of patients are 782 , 971 , and 973 on days 30,60, and 90, respectively. This corresponds to approximately $2.3 \%$ of the total population.

Estimating Hospital Utilization during an Unmitigated Epidemic

There are 33 facilities without hospitals within 10 miles of the ICE facility while all facilities have a hospital within 50 miles. A COVID-19 outbreak at a number of detention facilities would potentially overwhelm local ICU capacity within a 10- and 50-mile radius of each facility (Table 2). Under the most optimistic scenario ( $\mathrm{R} 0=2.5)$, and imagining synchronized epidemics across all facilities, estimated median aggregate ICU admissions from 56 facilities by day 30, 62 facilities by day 60 , and 65 facilities by day 90 would exceed ICU capacity within 10 miles of the facility. These numbers include those facilities with no ICU capacity within 10 miles. Under the least optimistic scenario $(\mathrm{R} 0=7)$, the numbers of facilities where ICU beds within 10 miles would not be adequate increases to 66 facilities within 30 days of onset and this persists through 60 and 90 days. A small number of facilities are also estimated to generate more ICU needs than ICU beds within 50 miles during the first 30 days of onset (six such facilities for
Table 2 Number of ICE facilities with an overwhelmed hospital catchment and additional ICU beds needed to meet ICE demand, by $\mathrm{R} 0$ and size of facility. This information is presented for days 30,60 , and 90 after the start of an outbreak

\begin{tabular}{lccc}
\hline Hospital catchment & 30 days & 60 days & 90 days \\
\hline ICE facilities where ICU bed demand exceeds capacity, R0 $=2.5$ \\
10 miles & 56 & 62 & 65 \\
50 miles & 6 & 7 & 8 \\
ICE facilities where ICU bed demand exceeds capacity, R0 $=3.5$ \\
10 miles & 58 & 66 & 66 \\
50 miles & 6 & 8 & 10 \\
ICE facilities where ICU bed demand exceeds capacity, R0 07.0 \\
10 miles & 66 & 66 & 66 \\
50 miles & 8 & 10 & 10 \\
\hline
\end{tabular}

$\mathrm{R} 0=2.5$ or 3.5 and eight such facilities for $\mathrm{R} 0=7$ ). For $\mathrm{R} 0=2.5$ and $\mathrm{R} 0=7$, the aggregate number of patients requiring ICU admission exceeds available beds within 50 miles for 8 and 10 facilities at 90 days, respectively.

\section{Discussion}

To our knowledge, this is the first analysis assessing the impact of COVID-19 on ICE detainees and the wider communities likely to care for detainees. Across the 111 ICE facilities we examined, and considering all three scenarios, the cumulative number of ICU admissions by day 90 for over half of facilities would exceed hospital capacity within a 10-mile radius. The impact on hospital capacity is lower if the radius was expanded to 50 miles, hovering around $8 \%$ of facilities exceeding ICU demand. These models do not take into account other concerns that would strain ICE's operational capacity at a time when staffing is likely to be a concern, such as the difficulty and risk to staff of repeatedly transporting and guarding detainees up to 50 miles distant from their facilities of origin. The timing and peak number of cases are driven by facility size, which also are important when considering preventive interventions.

Our findings suggest several possible interventions. Short of vaccination or a breakthrough treatment, effective interventions pertain to strategies to diffuse density. Lowering a facility's population density can slow the spread by lowering its R0. Figure S1 in the Supplementary Materials demonstrates the impact of doing so: the total number of infections drop, the time to peak infection shifts, and the 
proportion of the population infected is lower. This suggests two strategies for selective release: (1) release of detainees with chronic health conditions who are older in age and could be better cared for in a community setting or (2) release of detainees that are younger in age (under 35 years old) plus those with chronic health conditions regardless of age. The former would be a more limited release, while the latter would considerably enlarge the number of individuals released. Deportation through formal court processes - rather than release - may be a pathway to reducing the susceptible population in the USA. Given the current and growing regime of border closures, as well as the time, process, and number of contacts and transports necessary to deport rather than liberate a detainee at this time, such a strategy would not be without serious complications and risks to ICE personnel, US border patrol, the courts, and the detainees themselves. This is compounded by both human rights concerns about returning detainees to countries of origin that are dangerous or oppressive. Such complications to a strategy of mass deportation may render this approach less viable than release into the general domestic community. While liberating most or all of the individuals currently being held would add individuals susceptible to complications to the broader population, it is a less infectious environment than a detention facility. In addition, ICE could reaffirm its March 18, 2020 commitment to stop apprehending all but the most dangerous people it targets for immigration enforcement, thereby reducing the number of new lodgings introduced to their facilities [24].

Interventions within ICE facilities pose significant challenges, and reducing their R0 while maintaining detainee populations would require changes to a facility's physical space that may not be possible. The CDC [25, 26] and the Federal Bureau of Prisons [27] outline best practices for reducing the spread of infectious diseases in correctional settings, such as careful attention to hygiene and disinfecting surfaces, prompt quarantine, isolation and treatment measures, awareness and mitigation of the risks of physical contact between persons, and designing daily routines and systems that minimize interpersonal interaction in the event of outbreaks. It is unknown if distribution of PPE and strict hand washing protocols, for example, could help slow transmission (i.e., lower the R0) nor how quickly this could improve matters. Even when aligned with published standards, detention facilities are typically overcrowded [28], house multiple individuals in bunk bed settings, and offer limited potential for social distancing, reducing the effectiveness of these measures even when they are carefully followed. The recommended six feet of separation for social distancing necessitates an approximate $28 \mathrm{sq}$. foot area for each detainee; however, this exceeds the minimum 25 sq. foot standard published by the American Correctional Association. Many ICE holding areas are not able to meet that standard, and some only afford detainees 3 sq. feet of space in holding areas [29]. To at least some extent, this crowding is by design: the emphasis at these facilities is on behavior regulation techniques of control and surveillance made possible by a high population density and lower operating costs by minimizing the size of the facilities necessary to carry out this function [30]. Regardless of what the actual R0 of COVID-19 in ICE detention facilities turns out to be, it is likely to be higher than returning the same population of individuals to the wider community.

This analysis has several important limitations. The data used to populate the models were the most complete and up to date that could be obtained at the time of analysis. A crucial limitation is our reliance upon estimates of the proportions of individuals requiring hospitalization and ICU access, across different ages, reported by a CDC report based on limited data: in time, these proportions may prove to be overestimates and the calculations we provide seen as overestimates. There are no known estimates of R0 for COVID-19 in a given prison or detention population, so we relied on an analogous population. To account for this imprecision, we modeled three plausible R0 estimates, elevating the likelihood that the system's actual R0 was modeled within this range. Our estimates of eventual prevalence for a confined population substantially exceed available data from cruise ship and navy ship outbreaks but are very much in line with recent reports from a prison setting. Full analysis of data from confined populations will be an excellent topic for full retrospective analysis. The treatment capacity of local hospitals was calculated using data made public the first week of April, but actual capacity over time will vary depending on the rate at which the virus spreads in the general population, and we may therefore have underestimated need. While data reporting the current number of ICE detainees may differ from those used in this analysis, they lack the facility-level specificity needed to calculate ICU bed demand. For example, news reports indicate that ICE bookings have decreased and summary deportations after attempting to enter the USA have reportedly increased as a result of the COVID-19 pandemic [31]. If 
these data were publicly available at the facility level, our results could be rendered more precise. A dearth of similar studies resulted in the need to approximate key values. The actual age distribution of ICE detainees at each facility is unknown, and we relied on aggregate estimates from 2019 [19]. We assumed a fixed contact rate over the course of the epidemic; a variable rate may be more appropriate, especially if ongoing studies derive a variable rate specific to the COVID-19 epidemic. Our model included counties in their entirety if any part of them fell within the specified radii of 10 and 50 miles, so it is likely that community health care capacities within these radii are overestimated. We note that our key assumption in determining how many ICE facilities would overwhelm hospital ICU capacity within a 10and 50-mile radius was that all available ICU beds in these hospitals would be allocated to detainees in need of care. Overall, these assumptions suggest that our findings may have underestimated the number of infections and the number of facilities that will overwhelm the community ICU capacity.

Our results assume a static population within ICE facilities. In reality, detainees enter and depart the facilities and are moved between them. Given high rates of infection, especially among economically disadvantaged populations, in the general population of the USA, it is unclear whether ingress/egress of detainees would typically represent a source or a sink of infection from ICE facilities. To study this in full would require extensive data from ICE, coupled to a model of community transmission across the country, and is beyond the scope of our current work.

Finally, we presented the results here where all outbreaks were synchronized to start on the same day. There would likely be variation in the date of initial case within each facility, and subsequent peaks would potentially be distributed over a long time period. However, the analysis compared each facility's hospital needs with their corresponding community's ability to meet them using estimates that would not change based on such dates. Asynchronicity would principally mean that the model's results would not aggregate as demonstrated, but the individual facility-level analyses would still be illustrative of local challenges no matter how they were timed relative to those of other facilities.

The public health implications of this study are critical. They suggest that decisive action on the part of ICE will not only reduce morbidity and mortality outcomes in its population of detained immigrants but also minimize negative health outcomes in the communities that support ICE's detention facilities with health care resources. If hesitation prevails instead, and more limited measures on the part of ICE prove ineffective, then the successful social distancing strategies implemented in a community may be undone by the large number of detainee infectious disease cases that its hospitals must care for.

The study's other public health implications will endure beyond a pandemic which has brought the long-standing vulnerabilities of the nation's undocumented population into clearer relief. The COVID-19 pandemic highlights the need for further research into the health of the nation's undocumented immigrants and those detained by ICE. Beyond disaster planning, taking steps to improve the health and well-being of underserved populations is fundamental to public health.

Acknowledgments This study was funded by grants from the National Institute of General Medical Sciences (P20GM125507) and the National Institute on Drug Abuse (2T32DA01391116A1). We are grateful for the assistance of Ashley Truong in data compilation and preparation of this manuscript.

\section{Compliance with Ethical Standards}

Disclaimer The funding organizations had no role in the design and conduct of the study; in the collection, analysis, and interpretation of the data; and in the preparation, review, or approval of the manuscript.

\section{References}

1. Kassie E. Detained: how the US built the world's largest immigrant detention system. The Guradian Available at: https://www.theguardian.com/us-news/2019/sep/24 /detained-us-largest-immigrant-detention-trump. Accessed 16 April 2020.

2. Alvarez P, Shoichet CE. First ICE detainee tests positive for coronavirus. Available at: https://www.cnn.com/2020/03 /24/us/ice-detainee-coronavirus/index.html. Accessed 15 April 2020.

3. U.S. Immigration and Customs Enforcement. ICE guidance on COVID-19. Available at: https://www.ice. gov/coronavirus. Accessed 16 April 2020.

4. Ellis B, Hicken M. Medical care in immigrant detention centers under fire. Available at: https://www.cnn.com/2019 /10/04/us/immigrant-medical-care-wellpath-invs/index. html. Accessed 16 April 2020.

5. Shoichet C, Bracho-Sanchez E. These doctors risked their careers to expose the dangers children face in immigrant family detention. $C N N$. Available at: https://www.cnn. com/2019/05/23/health/ice-family-detention- 
whistleblowers-doctors/index.html. Accessed 16 April 2020.

6. American Immigration Council. Complaint demands investigation into inadequate medical and mental health care condition in immigration detention center. Available at: https://www.americanimmigrationcouncil.org/advocacy/ immigrants-inadequate-medical-care-aurora. Accessed 16 April 2020.

7. Homeland Security Office of Inspector General. Concerns about ICE detainee treatment and care at four detention facilities. 2019. Available at: https://www.oig.dhs. gov/sites/default/files/assets/2019-06/OIG-19-47-Jun19. pdf. Accessed 15 April 2020.

8. Ortega AN, McKenna RM, Kemmick Pintor J, et al. Health care access and physical and behavioral health among undocumented Latinos in California. Med Care. 2018;56(11): 919-26.

9. Beck TL, Le T-K, Henry-Okafor Q, Shah MK. Medical care for undocumented immigrants: national and international issues. Primary Care. 2017;44(1):e1-e13.

10. Carter JG, Mohler G, Ray B. Spatial concentration of opioid overdose deaths in Indianapolis: an application of the law of crime concentration at place to a public health epidemic. $J$ Contemp Crim Justice. 2019;35(2):161-85.

11. Volpenhein S, Candisky C (2020) Inmates, staff worry about care as Marion prison becomes one of largest outbreaks in U.S. Marion Star. Available at: https:/www.marionstar. com/story/news/local/2020/04/20/marion-county-ohiocoronavirus-prison-outbreak-largest-us/5166499002/. Accessed 20 April 2020.

12. Cases surge in an Ohio prison, making it a top U.S. hot spot. The New York Times; 2020.

13. Centers for Disease Control and Prevention. Hospitalization, intensive care unit (ICU) admission, and case-fatality percentages for reported COVID-19 cases, by age groupUnited States, February 12-March 16, 2020. MMWR Morb Mortal Wkly Rep. 2020;69(12):343-6.

14. Zhang S, Diao M, Yu W, Pei L, Lin Z, Chen D. Estimation of the reproductive number of novel coronavirus (COVID19) and the probable outbreak size on the diamond princess cruise ship: a data-driven analysis. Int J Infect Dis. 2020;93: 201-4.

15. Rocklöv J, Sjödin H, Wilder-Smith A. COVID-19 outbreak on the Diamond Princess cruise ship: estimating the epidemic potential and effectiveness of public health countermeasures. J Travel Med. 2020.

16. Backer JA, Klinkenberg D, Wallinga J. Incubation period of 2019 novel coronavirus (2019-nCoV) infections among travellers from Wuhan, China, 20-28 January 2020. Euro Surveill. 2020;25(5)

17. Wölfel R, Corman VM, Guggemos W, et al. Virological assessment of hospitalized patients with COVID-2019. Nature. 2020.

18. TRAC Immigation. About the data - ICE detention. Available at: https://rac.syr.edu/phptools/immigration/ detention/about_data.html. Accessed 12 April 2020.

19. Freedom for Immigrants. Detention by the numbers. Available at: https://www. freedomforimmigrants. org/detention-statistics. Accessed 17 April 2020.
20. Definitive Healtcare USA. Definitive healthcare bed locations ISO-19139 metadata. Available at: https://services7. arcgis.com/LXCny1HyhQCUSueu/arcgis/rest/services/ Definitive_Healthcare_USA_Hospital_Beds/FeatureServer/ $0 /$ metadata? format $=$ default $\& \mathrm{f}=\mathrm{html}$. Accessed $31 \mathrm{March}$ 2020 .

21. Definitive Healthcare. COVID-19 capacity predictor. Available at: https:/www.definitivehc.com/resources/ covid-19-capacity-predictor. Accessed 15 April 2020.

22. Unites States Military Health System. Zip Code Catchment Query Tool. Available at: https://health.mil/Military-HealthTopics/Technology/Support-Areas/Geographic-ReferenceInformation/Zip-Code-Catchment-Query-Tool. Accessed 15 April 2020.

23. United States Government Accountability Office. Immigration enforcement: arrests, detentions, and removals, and issues related to special populations. Vol GAO-20-36. Washington, D.C., USA: GAO; 2019.

24. Sacchetti M, Hernández A. ICE to stop most immigration enforcement inside U.S., will focus on criminals during coronavirus outbreak. 2020. Available at: https://www. washingtonpost.com/national/ice-halting-mostimmigration-enforcement/2020/03/18/d0516228-696c-11 ea-abef-020f086a3fab_story.html. Accessed 20 April 2020.

25. Centers for Disease Control and Prevention. Resources for correctional and detention facilities: before and during an outbreak. Available at: https://www.cdc.gov/coronavirus/ 2019-ncov/community/correction-detention/index.html. Accessed 16 April 2020.

26. Centers for Disease Control and Prevention. Interim guidance on management of coronavirus disease 2019 (COVID19) in correctional and detention facilities. Available at: https://www.cdc.gov/coronavirus/2019-ncov/community/ correction-detention/guidance-correctional-detention.html. Accessed 15 April 2020.

27. Federal Bureau of Prisons. Health management resources: clinical guidelines. Available at: https://www.bop. gov/resources/health_care_mngmt.jsp. Accessed 15 April 2020.

28. Dolovich S. Prison conditions: eScholarship, University of California; 2018.

29. Chalabi M. How severe is overcrowding at some US migrant facilities? Very. The Guardian. 2019. Available at: https://www.theguardian.com/us-news/2019/jul/05/usmigrant-facilities-severe-overcrowding. Accessed 20 April 2020 .

30. Jewkes Y. The aesthetics and anaesthetics of prison architecture. In: Simon J, Temple N, editors. Architecture and justice. New York: Routledge; 2013. p. 33-46.

31. Owen Q. Immigrant detention numbers decline as COVID19 spikes and controversial border policies take hold. 2020. Available at: https://abcnews.go.com/Politics/immigrantdetention-numbers-decline-covid-19-spikescontroversial/story?id=70094940. Accessed 18 April 2020.

Publisher's Note Springer Nature remains neutral with regard to jurisdictional claims in published maps and institutional affiliations. 